\title{
A safe "opioid" - is dry needling an efficacious alternative to opioids?
}

\begin{abstract}
Traditional Chinese medicine has used acupuncture for more than 2,000 years as a method to effectively treat numerous illnesses and diseases. It is in the Western model of medicine that acupuncture is considered 'alternative' medicine. The practice of this Western model of acupuncture, known as dry needling (DN), requires a technique that uses a monofilament without an injectate that is inserted into the soft tissue at varying depths-from superficial to deep-triggering a physiological response. Certain physicians and physical therapists have adopted this westernized version of the Chinese medicine modality by using the same technique for acupuncture to perform DN and electric dry needling (EDN). DN and EDN are administered to mediate analgesia peripherally, spinally, and supraspinally via multiple pathways by arousing the hypothalamic-pituitary-adrenal axis, provoking immune cells, and prompting the supraoptic nucleus. Stimulating these sites initiates the opioid interneuron mechanism resulting in opioid-based pain reduction that is mediated by the sympathetic nervous system and endogenous cannabinoids. Although EDN is further correlated with the spinal to supraspinal mechanisms of anti-nociception, both DN and EDN have shown to be components of a comprehensive analgesia pathway that acts like opioids in the human body. When comparing DN and EDN to opioid usage for analgesia, the adverse reactions and the significance of higher adverse reactions in the patient are essential factors to consider; DN and EDN have markedly less adverse effects than opioid use. They show high efficacy for analgesia and operate via similar mechanism of action as opioids. Therefore, the medical community should consider and utilize DN and EDN as an alternative to opioids in certain conditions.
\end{abstract}

Keywords: acupuncture, analgesia, dry needling, electric dry needling, hyperalgesia, morphine, opioid receptors, opioids
Volume 12 Issue I - 2019

\author{
Rashad Roberson, ${ }^{1,2}$ Britney Tucker, \\ Nicholas A Kerna ${ }^{2,3}$ \\ 'R\&R Rehab Associates, USA \\ ${ }^{2}$ College of Medicine, University of Science, Arts and Technology, \\ Montserrat, BWI \\ ${ }^{3}$ Department of CAM and Tropical Medicine, Suriwongse \\ Medical Center, Thailand
}

Correspondence: Nicholas A Kerna, College of Medicine, University of Science,Arts and Technology, 7583 Sourdough Drive, Morrison, CO 80465, USA, Email nicholas.kerna@usat.edu

Received: May 24, 2018 | Published: January 04, 2019
Abbreviations: ACTH, adrenocorticotropic hormone; AMPA, $\quad \alpha$-amino-3-hydroxy-5-methyl-4-isoxazolepropionic acid; ADP, adenosine diphosphate; ATP, adenosine triphosphate; COX-2, cyclooxygenase-2; CRH, corticotropin-releasing hormone; DN, dry needling; DRG, dorsal root ganglia; EDN, electric dry needling; NK1, neurokinin 1; NMDA, N-methyl-D-aspartate; OIH, opioid-induced hyperalgesia; ORL1, opioid receptor-like; SP, substance P; TRPv1, transient receptor potential vanilloid receptor subfamily1

\section{Introduction}

Opioids are some of the earliest known drugs used to treat pain, depression, insomnia, and anxiety. Opioids act on opioid receptors to produce morphine-like effects in the human body. However, the side effects of opioid use can include nausea, constipation, vomiting, itching, respiratory depression, addiction, tolerance, and opioidinduced hyperalgesia $(\mathrm{OIH})$. Although most side effects of prescribed opioid use are considered mild, a dangerous side effect is respiratory depression.

Opioid receptors are found throughout the spine, central nervous system, and gastrointestinal tract and mediate morphine-like effects. In the 1960s, the mechanisms of opiates were found to be mediated by multiple types of molecular receptors throughout the body. The three classic opioid receptors that mediate analgesia are norciceptors. Tolllike receptor 4, and sigma receptors. Sigma receptors are no longer considered opioid receptors in that they are not reversible when opioid antagonists, such as Naloxone or Naltrexone, are given. ${ }^{1}$

The opioid receptor classes mu, kappa, and delta are the top classes of opioid receptors that are used due to their high affinity for binding to the opioid binding sites and are stereoselective for levorotary isomers. The mu opioid receptor (which has three subtypes) and the opioid receptor-like (ORL1) receptor are clinically relevant in pain analgesia. These opioid receptors are G-protein coupled receptors involved in GABA-ergic neurotransmission.

The pharmacodynamics of opioids correlate with the class of receptors they bind to, the affinity they have for the receptors, and whether they are antagonist or agonist. Various opioid receptors will stimulate a variety of opioid effects; for instance, the mu-1 subtype will result in supraspinal analgesia, the mu-2 subtype will result in respiratory depression and dependence, and the kappa subtype will result in spinal analgesia and sedation.

It is via these same mechanisms of action that DN and EDN influence analgesia. DN and EDN affect supraspinal and spinal analgesia by the stimulation of opioid receptors without using an injectate. With DN and EDN, eliciting analgesia is not limited to the periphery; and in decreasing second-order neuron hyperalgesia, perception of pain at the central nervous system is decreased. Thus, an opioid-like mechanism is recognized. This opioid-like mechanism of action allows DN and EDN to be considered as a substitute for opioid use with the benefit of lessening opioid addiction. 


\section{Discussion}

Addressing the pathways of anti-nociception that utilize opioid mechanisms of analgesia is the focus of this discussion. Centrally mediating pain is accomplished by nociceptors that are bipolar: starting with the cell body at the dorsal root ganglion (DRG), one nerve ending going toward the periphery, the other end going into the dorsal column to communicate with second order neurons. The $\mathrm{C}$-fibers initiate the communication in the DRG and trigger the release of glutamate and Substance P (SP). This release of SP, in turn, triggers second-order $\quad \alpha$-amino-3-hydroxy-5-methyl-4-isoxazolepropionic acid (AMPA) receptor via phosphorylation as the glutamate release is sustained. It requires activation of neurokinin-1 (NK1) second-order receptors via SP to cause phosphorylation of N-methyl-D-aspartate (NMDA) second-order receptors. The phosphorylation of NMDA receptors creates an influx of calcium ions $(\mathrm{Ca} 2+)$ across the membrane leading to the up-regulation, awakening, and sensitization of NMDA receptors, and the up-regulation of cyclooxygenase-2 (COX-2) enzyme that increases the production of prostaglandins. Furthermore, the repeated channel use of NMDA and AMPA receptors results in cell death at the postsynaptic neuron and a cytotoxic environment that elicits interneuron apoptosis of the dorsal horn. ${ }^{1}$

The dorsal horn interneurons inhibit the afferent receptive field. When the dorsal horn is disinhibited, the nociceptive afferents that were latent can stimulate the dorsal horn using synapses that were previously not used. ${ }^{1}$ This process results in an increased receptive field peripherally and is the basis of referred pain; in which the expression of pain via the spinothalamic tract is termed, hyperalgesia. The release of glutamate, SP, and adenosine triphosphate (ATP) also stimulates glial cells and astrocytes in the dorsal horn giving rise to spinal hyperalgesia. Central sensitization is the result of hyperalgesia peripherally and spinally. Multiple studies describe central sensitization with low back and shoulder fibromyalgia due to increased sensitivity to mechanical stimulation and receptor field expansion in the dorsal horn in relation to pain threshold. ${ }^{1}$

Goldman et al. $(2010)^{2}$ found a significant release of ATP, adenosine diphosphate (ADP), and adenosine in the interstitial fluid after DN for 30 minutes at acupuncture point ST36; most likely due to stimulation of the transient receptor potential vanilloid receptor subfamily 1 (TRPv1) receptors following calcium wave propagation. ${ }^{2,3}$ ATP was then catabolized to adenosine and, in turn, activated A1 adenosine receptors. ${ }^{3}$ Research revealed that mice experiencing inflammatory or neuropathic pain had normal A1 adenosine receptors showing less activation of the cingulate gyrus versus mice that had A1 adenosine receptors blocked; the cingulate gyrus is the emotional center in the brain for pain experience. ${ }^{4}$ A1 receptors, which are G-coupled protein receptors, are found on the afferent DRG; the nerve endings possibly function by blocking adenylyl cyclase. Thus, the stimulation of A1 receptors via DN-mediated analgesia is comparable to opioids blocking adenylyl cyclase in opioid analgesia.

Studies have indicated that EDN only affects the purinergic receptors $\mathrm{P} 2 / \mathrm{X} 2$ and $\mathrm{P} 2 / \mathrm{X} 3$ in neuropathic pain. ${ }^{5}$ Mice that were inflicted, bilaterally, with hind leg injuries showed an elevation of these purinergic receptors, increased notably at the DRG L4/L5. ${ }^{6}$ Application of EDN, bilaterally, at acupuncture points ST36 and GB34 for seven days at 30 minutes per day dramatically decreased purinergic receptors, increased ATP, and improved mechanical pain threshold. ${ }^{6,7}$ Although direct opioid analgesic pathway mechanisms were not in play, desensitization of spinal pain pathways, lessened supraspinal communication, and diminished perception centrally of pain did result (as occurs with opioids).

In the neuroendocrine system, there was an increase in activity of the hypothalamic-pituitary-adrenal axis with inflammation in humans and animals. There was a significant increase in all hormones in that pathway-from corticotropin-releasing hormone (CRH) to adrenocorticotropin hormone (ACTH) to cortisol-in mice as inflammation occurred, but not so when void of inflammation. ${ }^{5}$ Beta-endorphins were increased at the paraventricular nucleus. ${ }^{6,7}$ The released endogenous opioids attached to opioid receptors resulting in analgesia. This pathway may have a complementary effect on the opioid receptors in humans. Again, it was shown that opioid pathways were stimulated with DN and EDN.

The CRH-POMC-corticosteroid axis is triggered by mechanical stress via human dermal fibroblasts. ${ }^{8}$ Dermal fibroblasts can produce $\mathrm{CRH}$ and express $\mathrm{CRH}$ receptors. ${ }^{1,6}$ When inflammation occurs, $\mathrm{CRH}$ influences opioid release from immune cells enhancing EDN-mediated analgesia. Conversely, EDN-mediated analgesia is inhibited and decreased when CRH antagonists are used..$^{9}$ There is a descending pain modulation system that increases the concentration of opioids, norepinephrine, and serotonin in the spine due to EDN, while it decreases the concentration of norepinephrine in the brain due to stimulation of the arcuate nucleus and periaqueductal gray with EDN. ${ }^{9}$ When the arcuate nucleus and periaqueductal gray are stimulated, enkephalins and dynophins are released. The use of an opioid antagonist, such as Naloxone, limits DN-mediated analgesia. ${ }^{10}$ A2-adrenergic receptors and 5-HT receptors on enkephalinergic interneurons in the dorsal column encourage the release of enkephalins. Enkephalins recognize three classes of opioid receptors (mu, kappa, and delta receptors) which are presynaptic and postsynaptic. Thus, encephalins affect pathways altering the transmission of pain through the spinothalamic tract decreasing supraspinal perception of pain. All three receptors are stimulated during acute pain, via the enkephalins, but only two receptors are stimulated during chronic pain. ${ }^{11}$ These tracts, as mentioned above, are a direct indication of DN-mediated analgesia.

\section{Conclusion}

Opioids can relieve certain degrees of discomfort or pain resulting from injury, illness or surgery; but opioid addiction and abuse has become an epidemic, perhaps doing more harm than good. The use of an alternative method of treatment, in some cases, can aid in dramatically reducing the use of opioids. Acupuncture is a time-tested method that has influenced the development of DN and EDN to elicit a morphine-like effect without the harmful side effects of certain narcotics. DN and EDN seem to be effective methods that allow patients to return to activities rather than stay sidelined by chronic pain and adverse effects of opioids. DN and EDN are cost-effective and less harmful to the patient in that they allow the body to provide its pain relief. Dry needling appears to be a safe and efficacious alternative to opioids. DN, as an alternative to opioids, helps mitigate opioid addiction and other unwanted opioid side effects.

\section{Acknowledgments}

T.L. Brown (Harvard University) is acknowledged for his early contribution to this project. 


\section{Conflicts of interest}

The authors declare that the research was conducted in the absence of any commercial or financial relationship that could be construed as a conflict of interest.

\section{References}

1. Dunning J, Butts R, Mourad F, et al. Dry needling: a literature review with implications for clinical practice Guidelines. Phys Ther Rev. 2014;19(4):252-265.

2. Goldman N, Chen M, Fujita T, et al. denosine A1 receptors mediate local anti-nociceptive effects of acupuncture. Nat Neurosci. 2010;13(7):883888 .

3. Butts RJ, Dunning J, Grubb MS, et al. Peripheral and Spinal Mechanisms of Pain and Dry Needling Mediated Analgesia: A Clinical Resource Guide for Health Care Professionals. International Journal of Physical Medicine \& Rehabilitation. 2016.

4. Tu WZ, Cheng RD, Cheng B, et al. Analgesic effect of electroacupuncture on chronic neuropathic pain mediated by $\mathrm{P} 2 \mathrm{X} 3$ receptors in rat dorsal root ganglion neurons. Neurochem Int. 2012;60(4):379-386.

5. Park JY, Park JJ, Jeon S, et al. from peripheral to central: the role of ERK signaling pathway in acupuncture analgesia. J Pain. 2014;15(5):535549 .
6. Pinheiro AR, Paramos-de-Carvalho D, Certal M, et al. Histamine induces ATP release from human subcutaneous fibroblasts, via pannexin-1 hemichannels, leading to $\mathrm{Ca}+2$ mobilization and cell proliferation. $J$ Biol Chem. 2013;288(38):27571-27583.

7. Canadian Gynecology Institute of Chinese Medicine (CGICM). (2013). Retrieved from http://cgicm.ca/wp/wp-content/uploads/2013/06/acurotating.jpg

8. Wu SY, Chen WH, Hsieh CL, et al. Abundant expression and functional participation of TRPV1 at Zusanli acupoint (ST 36) in mechnaosensitive TRPV1 as an acupuncture- responding channel. BMC Complement Altern Med. 2014;14:96.

9. Hsieh YL, Chou LW, Joe YS, et al. Spinal Cord mechanism involving the remote effects of dry needling on the irritability of myofascial trigger spots in rabbit skeletal muscle. Arch Phys Med Rehabil. 2011;92(7):1098-1105.

10. Jafri MS. Mechanisms of Myofascial Pain. Int Sch Res Notices. 2014;523924.

11. Gerber LH, Shah J, Rosenberger W, et al. Dry Needling Alters Trigger Points in the Upper Trapezius Muscle and Reduces Pain in Subjects With Chronic Myofascial Pain. PM R. 2015;7(7):711-718. 